EESTI NSV TEADUSTE AKADEEMIA TOIMETISED. X KÖIDE

FOOSIKALIS-MATEMAATILISTE JA TEHNILISTE TEADUSTE SEERIA. 1961, NR. 2

ИЗВЕСТИЯ АКАДЕМИИ НАУК ЭСТОНСКОЙ ССР. ТОМ Х СЕРИЯ ФИЗИКО-МАТЕМАТИЧЕСКИХ И ТЕХНИЧЕСКИХ НАУК, 1961, № 2

\title{
ОБ ОПТИЧЕСКИХ ЯВЛЕНИЯХ ПРИ НАКЛОННОМ ПРОСВЕЧИВАНИИ ФОТОУПРУГИХ МОДЕЛЕЙ
}

\author{
Х. АБЕН, \\ кандидат технических наук
}

\section{I. ВВЕДЕНИЕ}

Наклонное просвечивание является эффективным методом разделения главных напряжений при исследовании плоского напряженного состояния методом фотоупругости; в случае же объемного напряженного состояния (при исследовании замороженных срезов) наклонное просвечивание позволяет определять пять компонентов тензора напряжения.

Во избежание преломления поляризованного света при наклонном падении на поверхность моделей, их обычно погружают в иммерсионную жидкость, имеющую такой же показатель преломления, как и материал модели. Применение иммерсионной жидкости значительно осложняет проведение эксперимента и часто связано с большими техническими трудностями. То же относится и к применению вспомогательных призм специальной формы [1,2].

Хотя при непосредственном наклонном просвечивании изображение модели получается несколько более сжатым, чем при погружении в иммерсионную жидкость (при равных углах преломления), проведение эксперимента при этом значительно проще. Некоторыми исследователями уже использовалось непосредственное наклонное просвечивание, однако в литературе отсутствует теоретическое обоснование этого метода. В настоящей статье рассматриваются оптические явления, возникающие при непосредственном наклонном просвечивании фотоупругих моделей, и выясняются вопросы интерпретации экспериментальных данных в этом случае.

Так как оптическая анизотропия фотоупругих моделей весьма малая, то будем рассматривать интересующие нас оптические явления на основе теории преломления света на границе двух изотропных диэлектриков. В этом случае преломление характеризуется следующими закономерностями (см. $\left.\left.{ }^{3,4}\right]\right)$.

1. Угол падения $i$ и угол преломления $r$ связаны соотношением

$$
\frac{\sin i}{\sin r}=\frac{n_{2}}{n_{1}}
$$

где $n_{1}, n_{2}$ - показатели преломления первой и второй среды.

2. Плоскополяризованный свет остается после преломления плоскополяризованным, при этом электрический вектор падающего света по- 
ворачивается в направлении плоскости падения. Пусть $\alpha_{0}-$ угол между электрическим вектором падающего света и плоскостью падения, $\alpha_{\mathbf{m}}$ - тот же угол преломленного света. Тогда

$$
\operatorname{tg} \alpha_{M}=\operatorname{tg} \alpha_{0} \cos (i-r) \text {. }
$$

3. Амплитуда электрического вектора преломленного света $E_{\mathrm{M}}$ выражается соотношением

$$
E_{\mathrm{M}}=E_{0} \frac{2 \cos i \sin r}{\sin (i+r) \cos (i-r)} \sqrt{1-\sin ^{2}(i-r) \sin ^{2} \alpha_{0}},
$$

где $E_{0}$ - амплитуда электрического вектора падающего света.

4. Фазы световых колебаний при преломлении не изменяются.

В дальнейшем будем рассматривать наклонное просвечиеание плоскопараллельной пластинки, которая в общем случае подвергнута объемному напряженному состоянию (например, исследование замороженного среза). Главные напряжения могут быть ориентированы произвольно.

\section{II. ПРИМЕНЕНИЕ ПОЛЯРИСКОПА, ПОЛЯРИЗАТОР И АНӒЛИЗАТОР КОТОРОГО НЕЗАВИСИМО ПОВОРАЧИВАЕМЫ}

\section{1. Определение квазиглавных направлений}

Вначале рассмотрим прохождение плоскополяризованного света через оптически изотропную плоскопараллельную пластинку. Пусть $P_{0}$ обозначает направление электрнческого вектора падающего света (фиг. 1), который составляет с плоскостью падения угол $\alpha_{0}$. При преломлении $P_{0}$ поворачивается в направлении плоскости падения на угол $\gamma_{0}$, который определяется соотношением (2). В пластинке электрический вектор $P_{м}$ составляет с плоскостью падения угол $\alpha_{м}=\alpha_{0}-\gamma_{0}$.

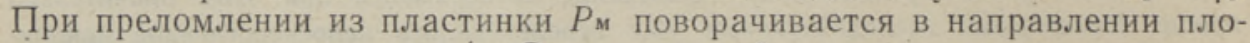
скости падения на угол $\gamma_{*}^{\prime}$. Электрический вектор выходящего света $P_{*}$ составляет с $P_{0}$ угол

$$
\gamma_{0}+\gamma_{*}^{\prime}=\alpha_{0}-\alpha_{*} .
$$

В фотоупругой модели $P_{\text {м }}$ разлагается на направления квазиглавных напряжений $\sigma_{1}^{\prime}, \sigma_{2}^{\prime}(x, y)$. Между колебаниями $x$ и $y$ возникает разность фаз, вследствие чего выходящий из модели свет в общем случае эллиптически поляризован.

Если же $P_{\mathrm{s}}$ совпадает с одним из квазиглавных направлений, то поляризация света при прохождении модели не изменяется и из модели выходит плоскополяризованный свет, направление электрического вектора которого можно определять анализатором. Таким образом, для определения квазиглавных направлений необходимо найти такое положение поляризатора, при котором выходящий из модели свет плоскополяризован. В этом случае $\alpha_{\mathrm{M}}=\beta$ (фиг. 1$)$, и угол между квазиглавным направлением и плоскостью падения $\beta$ можно определять из соотношения

$$
\operatorname{tg} \beta=\operatorname{tg} \alpha_{0} \cos (i-r)=\frac{\operatorname{tg} \alpha_{*}}{\cos (i-r)}
$$




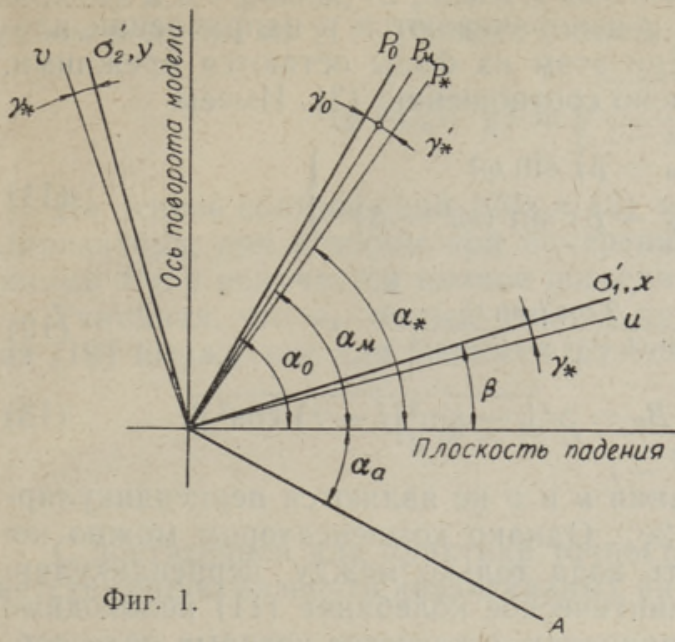

На основе формулы (2) можем определить угол $\gamma_{0}$

$$
\begin{aligned}
& \operatorname{tg} \gamma_{0}=\operatorname{tg}\left(\alpha_{0}-\alpha_{M}\right)= \\
= & \frac{\operatorname{tg} \alpha_{0}[1-\cos (i-r)]}{1+\operatorname{tg}^{2} \alpha_{0} \cos (i-r)} .
\end{aligned}
$$

Значения угла $\gamma_{0}$ при различных значениях $\alpha_{0}$ для случая $i=55^{\circ}, n=n_{2}=1,5\left(n_{1}=1\right)$ приведены в нижеследующей таблице:

\begin{tabular}{c|c|c|c|c|c|c|c|c|c|c}
\hline$a_{0}$ & $0^{\circ}$ & $10^{\circ}$ & $20^{\circ}$ & $30^{\circ}$ & $40^{\circ}$ & $50^{\circ}$ & $60^{\circ}$ & $70^{\circ}$ & $80^{\circ}$ & $90^{\circ}$ \\
\hline$\gamma_{0}$ & $0^{\circ} 00^{\prime}$ & $0^{\circ} 42^{\prime}$ & $1^{\circ} 20^{\prime}$ & $1^{\circ} 49^{\prime}$ & $2^{\circ} 06^{\prime}$ & $2^{\circ} 07^{\prime}$ & $1^{\circ} 53^{\prime}$ & $1^{\circ} 25^{\prime}$ & $0^{\circ} 46^{\prime}$ & $0^{\circ} 00^{\prime}$
\end{tabular}

Данные этой таблицы показывают, что при преломлении углы поворота двух перпендикулярных световых колебаний практически равны.

Из соотношения (6) получим, что угол $\gamma_{0}$ имеет максимальное значение в случае

$$
\operatorname{tg} \alpha_{0}=\frac{1}{\sqrt{\cos (i-r)}} .
$$

Если $i=55^{\circ}, n=1,5$, то из (7) имеем $\alpha_{0}=46^{\circ}$.

\section{2. Измерение разности хода}

Рассмотрим случай, когда плоскость падения и $P_{0}$ находятся относительно квазиглавных направлений в разных квадрантах (фиг. 1). Пусть $P_{0}$ выражается в виде

$$
P_{0}=I \sin \omega t
$$

где $I$ - амплитуда падающего света. После преломления имеем

$$
P_{\mathrm{M}}=I^{\prime} \sin \omega t,
$$

где $I^{\prime}$ определяется формулой (3).

Колебание $P_{\text {м }}$ разлагается на квазиглавные направления. Перед. выходом из модели колебания $x$ и $y$ выражаются в виде

$$
x=I^{\prime} \cos \left(\alpha_{\mathrm{M}}-\beta\right) \sin \omega t, \quad y=I^{\prime} \sin \left(\alpha_{\mathrm{M}}-\beta\right) \sin (\omega t-\Delta),
$$

где $\Delta=\frac{2 \pi}{\lambda} C t^{\prime}\left(\sigma_{1}^{\prime}-\sigma_{2}^{\prime}\right), \lambda$ - длина волны, $C$ - оптическая постоянная, $t^{\prime}=t / \cos r, t-$ толщина модели. 
Преломляясь, колебания $x$ и $y$ поворачиваются в направлении плоскости падения на оси $u$ и $v$. При этом их фазы остаются прежними, амплитуды же изменяются согласно соотношению (3). Имеем

где

$$
\left.\begin{array}{l}
u=I^{\prime \prime} B_{1} \cos \left(\alpha_{M}-\beta\right) \sin \omega t \\
v=I^{\prime \prime} B_{2} \sin \left(\alpha_{M}-\beta\right) \sin (\omega t-\Delta)
\end{array}\right\}
$$

Выходящие из модели колебания $u$ и $v$ не являются перпендикулярными, а составляют угол $90^{\circ}+2 \gamma_{*}$. Однако компенсатором можно непосредственно измерять разность хода только между перпендикулярными колебаниями. Поэтому эллиптическое колебание (11) необходимо описывать в прямоугольных координатах, в качестве которых целесообразно использовать оси $x$ и $y$.

Обозначаем колебания (11) сокращенно

$$
u=a \sin \omega t, \quad v=b \sin (\omega t-\Delta) .
$$

Проектируя $u$ и $v$ на оси $x$ и $y$, имеем

$x=u \cos \gamma_{*}-v \sin \gamma_{*}=a \cos \gamma_{*} \sin \omega t-b \sin \gamma_{*} \sin (\omega t-\Delta)$

$\left.y=-u \sin \gamma_{*}+v \cos \gamma_{*}=-a \sin \gamma_{*} \sin \omega t+b \cos \gamma_{*} \sin (\omega t-\Delta)\right\}$

и после преобразования

$$
\left.\begin{array}{l}
x=\left(a^{2} \cos ^{2} \gamma_{*}+b^{2} \sin ^{2} \gamma_{*}-2 a b \sin \gamma_{*} \cos \gamma_{*} \cos \Delta\right)^{1 / 2} \sin \left(\omega t-w_{1}\right) \\
y=\left(a^{2} \sin ^{2} \gamma_{*}+b^{2} \cos ^{2} \gamma_{*}-2 a b \sin \gamma_{*} \cos \gamma_{*} \cos \Delta\right)^{1 / 2} \sin \left(\omega t-w_{2}\right)
\end{array}\right\}
$$

где

$$
\begin{gathered}
\operatorname{tg} w_{1}=\operatorname{tg} \Delta \frac{1}{1-K \frac{\operatorname{ctg} \gamma_{*}}{\cos \Delta}}, \quad \operatorname{tg} w_{2}=\operatorname{tg} \Delta \frac{1}{1-K \frac{\operatorname{tg} \gamma_{*}}{\cos \Delta}} \\
K=\frac{a}{b} .
\end{gathered}
$$

Из выражений (17) находим разность фаз колебаний (16)

$$
\operatorname{tg} w=\operatorname{tg}\left(w_{2}-w_{1}\right)=\operatorname{tg} \Delta \frac{\cos 2 \gamma_{*}}{1-\frac{K^{2}+1}{2 K} \frac{\sin 2 \gamma_{*}}{\cos \Delta}} .
$$

Если оси компенсатора составляют с анализатором угол $45^{\circ}$ (диагональное положение), то для полного погасания света при компенсации необходимо, чтобы компенсируемые световые колебания имели равные амплитуды. Из соотношений (16) и (14) видно, что если амплитуды ко-

* Это обеспечивает наибольшую точность при измерении разности хода. 
лебаний $u$ и $v$ равны, то равны и амплитуды колебаний $x$ и $y$. Таким образом получим из (11) условие полной компенсации

$$
\operatorname{tg}\left(\alpha_{M}-\beta\right)=\sqrt{\frac{1-\sin ^{2}(i-r) \sin ^{2} \beta}{1-\sin ^{2}(i-r) \cos ^{2} \beta}} .
$$

На основе соотношений (20) и (2) можно определять положение поляризатора, при котором при измерении разности хода между колебаниями $x$ и $y$ получается полное погасание света.

Учитывая, что $\gamma_{*}$ малый угол, а при компенсации $K \approx 1^{\star}$, получим из (19) выражение для разности фаз колебаний $x$ и $y$

$$
\operatorname{tg} w=\operatorname{tg} \Delta \frac{1}{1-\frac{\sin 2 \gamma_{*}}{\cos \Delta}}
$$

С достаточной для практики точностью можно разность фаз $\Delta$, соответствующую разности квазиглавных напряжений, определять формулой

$$
\operatorname{tg} \Delta=\operatorname{tg} w\left(1-\frac{\sin 2 \gamma_{*}}{\cos w}\right)
$$

На фиг. 2 сплошной кривой показана зависимость $ш-\Delta$ от $\Delta$ по формуле (21) при $\gamma_{*}=2^{\circ} 07^{\prime}$.

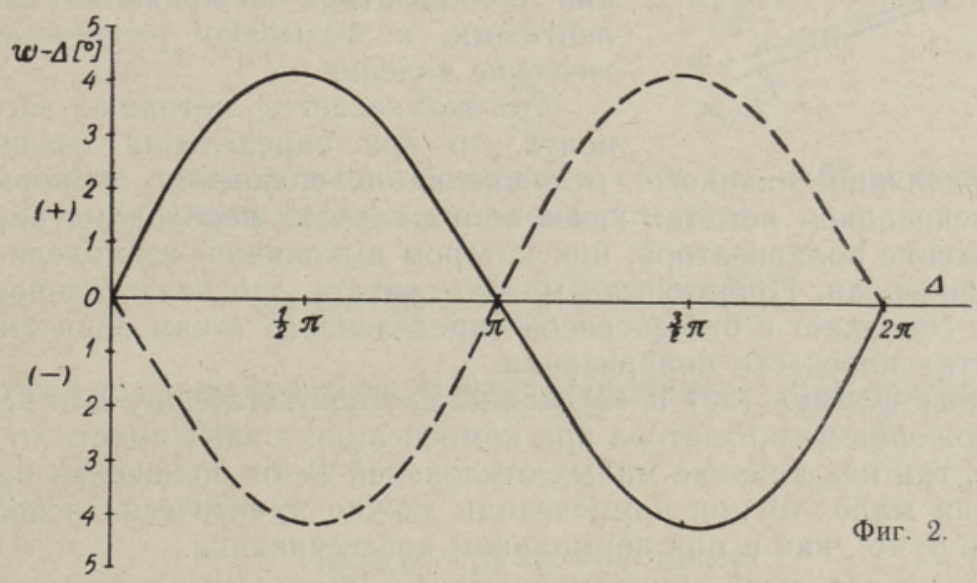

Если $P_{0}$ и плоскость падения находятся относительно квазиглавных направлений в одном и том же квадранте (рис. 3 ), то имеем

$$
u=I^{\prime \prime} B_{1} \cos \left(\alpha_{\mathrm{M}}+\beta\right) \sin \omega t, v=I^{\prime \prime} B_{2} \sin \left(\alpha_{\mathrm{M}}+\beta\right) \sin (\omega t-\Delta),
$$

откуда получим условие полной компенсации

$$
\operatorname{tg}\left(\alpha_{M}+\beta\right)=\sqrt{\frac{1-\sin ^{2}(i-r) \sin ^{2} \beta}{1-\sin ^{2}(i-r) \cos ^{2} \beta}} .
$$

В данном случае угол между световыми колебаниями при преломлении уменьшается, т. е. в формуле (21) угол $\gamma_{*}$ является отрицательным.

- При $i=55^{\circ}, \quad n=1,5: 0,92<K<1,08$. 
Для измеряемой разности фаз получим выражение

или

$$
\operatorname{tg} w=\operatorname{tg} \Delta \frac{1}{1+\frac{\sin 2 \gamma_{*}}{\cos \Delta}}
$$

$$
\operatorname{tg} \Delta=\operatorname{tg} w\left(1+\frac{\sin 2 \gamma_{*}}{\cos w}\right) .
$$

Зависимость $w-\Delta$ от $\Delta$ по формуле (25) показана на фиг. 2 пунктирной кривой.

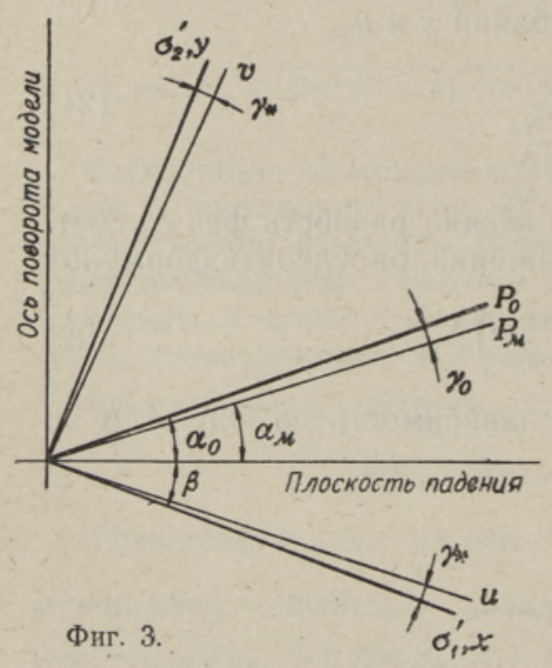

Таким образом, при наклонном просвечивании измеряемая разность фаз зависит не только от разности квазиглавных напряжений, но и от угла $\gamma_{*}$ и от положения электрического вектора падающего света. В зависимости от положения последнего и от величины $\Delta$ измеряемая разность фаз w больше или меньше разности фаз $\Delta$, непосредственно связанной с разностью квазиглавных напряжений. По-видимому, впервые при применении метода фотоупругости имеет значение положение электрического вектора, т. е. два перпендикулярных между собой положения поляризатора не являются эквивалентными, а вызывают различные оптические явления.

Что же касается методики эксперимента, то при определении квазиглав-

ных направлений никакого осложнения в сравнении с нормальным просвечиванием нет, так как в обоих случаях необходимо определять положение поляризатора, при котором выходящий из модели свет плоскополяризован. Практически можно считать, что квазиглавное направление совпадает с биссектрисой определенного таким образом «угла поворота» плоскости поляризации.

На основе формул (20) и (2) можно составить таблицу для нахождения положения поляризатора при компенсации в зависимости от угла $\beta$. Однако, так как влияние малых отклонений $P_{0}$ от положения полной компенсации мало, то при компенсации можно практически использовать $\alpha_{0}=\beta \pm 45^{\circ}$, как и при нормальном просвечивании.

Если величиной $\sin 2 \gamma_{*} / \cos w$ нельзя пренебречь, то легко получить уточненное значение $\Delta$ по формулам (22) или (26) *.

\section{III. ПРИМЕНЕНИЕ ПЛОСКОГО ПОЛЯРИСКОПА}

\section{1. Определение изоклин}

Для определения изоклины в скрещенном полярископе необходимо определять такое положение поляризатора, при котором интенсивность выходящего из анализатора света минимальна. Проектируя выходящие из модели колебания $u$ и $v(11)$ на направление анализатора и учитывая, что $\alpha_{\mathrm{a}}=90^{\circ}-\alpha_{\mathrm{m}}-\gamma_{0}$ (фиг. 1), имеем

* Если $\gamma_{*}=2^{\circ} 07^{\prime}$, то при $\lambda=589$ м $\mu$ поправка в разности хода может составить 7 м $\mu$. 
$A=u \cos \left(90^{\circ}+\beta-\alpha_{M}-\gamma_{0}-\gamma_{*}\right)-v \cos \left(\alpha_{M}-\beta+\gamma_{0}-\gamma_{*}\right)$.

Интуитивно вообразимо, что искомое положение поляризатора находится вблизи квазиглавного направления. Поэтому можем считать

$$
\gamma_{0}=\gamma_{*}=\gamma \text {. }
$$

Теперь находим на основе (27) и (11), учитывая малость угла $\gamma$,

$$
\begin{gathered}
A=I^{\prime \prime} B_{1}\left[\sin 2 \gamma \cos ^{2}\left(\alpha_{M}-\beta\right)+\sin \left(\alpha_{M}-\beta\right) \cos \left(\alpha_{M}-\beta\right)\right] \sin \omega t- \\
-I^{\prime \prime} B_{2} \sin \left(\alpha_{M}-\beta\right) \cos \left(\alpha_{M}-\beta\right) \sin (\omega t-\Delta) .
\end{gathered}
$$

Обозначая амплитуду колебания (29) $A_{1}$, получим из условия минимума $A_{1}^{2}$ по углу $\left(\alpha_{\mathrm{m}}-\beta\right)$

$$
\frac{\partial\left(A_{1}^{2}\right)}{\partial\left(a_{M}-\beta\right)}=0
$$

уравнение для определения угла $\left(\alpha_{M}-\beta\right)$

$$
\begin{aligned}
& 2\left(2 K_{1} \cos \Delta-K_{1}^{2}-1\right) \operatorname{tg}^{3}\left(\alpha_{M}-\beta\right)+6 K_{1} \sin 2 \gamma\left(\cos \Delta-K_{1}\right) \operatorname{tg}^{2}\left(\alpha_{M}-\beta\right)+ \\
& \quad+2\left(K_{1}^{2}-2 K_{1} \cos \Delta+1\right) \operatorname{tg}\left(\alpha_{M}-\beta\right)+2 K_{1} \sin 2 \gamma\left(K_{1}-\cos \Delta\right)=0,
\end{aligned}
$$

где

$$
K_{1}=\frac{B_{1}}{B_{2}} .
$$

На основе предположения о малости угла $\left(\alpha_{м}-\beta\right)$ можем в уравнении (31) отбросить первые два члена, что дает

$$
\operatorname{tg}\left(\alpha_{M}-\beta\right)=\frac{K_{1} \sin 2 \gamma\left(\cos \Delta-K_{1}\right)}{K_{1}\left(K_{1}-2 \cos \Delta\right)+1} .
$$

С достаточной для практики точностью можно в (33) считать $K_{1}=1$.

Имеем

$$
\operatorname{tg}\left(\alpha_{M_{i}}-\beta\right)=-\frac{1}{2} \sin 2 \gamma
$$

и далее

$$
\alpha_{M}-\beta=-\gamma
$$

Так как $\alpha_{0}=\alpha_{\mathrm{M}}+\gamma$, то интенсивность выходящего из анализатора света минимальна тогда, когда $\alpha_{0}=\beta$, т. е. поляризатор параллелен одному из квазиглавных направлений. Таким образом, определение изоклин при наклонном просвечивании никаких коррективов по сравнению с нормальным просвечиванием не требует.

При наклонном просвечивании интенсивность света на изоклине не равна нулю, а по (29) и (35) равна

$$
\frac{A_{1}^{2}}{\left(I^{\prime \prime}\right)^{2}}=\sin ^{2} \gamma\left(B_{1}^{2}+B_{2}^{2}+2 B_{1} B_{2} \cos \Delta\right) \text {. }
$$


Практически наибольшая интенсивность (при $i=55^{\circ}, \quad n=1,5$; $\cos \Delta=+1)$ составляет $A_{1}^{2} /\left(I^{\prime \prime}\right)^{2}=0,0049$. При определении изоклин по отдельным точкам (на приборе типа КСП-5) эта интенсивность не влияет заметно на точность эксперимента. При фотографировании изоклин это может несколько уменьшать их четкость.

\section{2. Измерение разности хода}

Разность хода целесообразно измерять на осях $x$ и $y$. Для совмещения осей компенсатора с осями $x$ и $y$ необходимо после определения изоклины поворачивать поляризатор на $45^{\circ}$, как и при нормальном просвечивании.

Учитывая, что в данном случае

$$
\alpha_{\mathrm{M}}-\beta=45^{\circ} \div \gamma_{0},
$$

колебания $u$ и $v(11)$ приобретают вид

$$
\left.\begin{array}{c}
u=I^{\prime \prime} B_{1} \cos \left(45^{\circ}-\gamma_{0}\right) \sin \omega t=C_{1} \sin \omega t \\
v=I^{\prime \prime} B_{2} \sin \left(45^{\circ}-\gamma_{0}\right) \sin (\omega t-\Delta)=C_{2} \sin (\omega t-\Delta)
\end{array}\right\}
$$

Проектируя колебания (38) на оси $x$ и $y$, имеем

$$
\left.\begin{array}{l}
x=\sqrt{C_{1}^{2}-2 C_{1} C_{2} \sin \gamma_{*} \cos \Delta} \sin \omega t \\
y=\sqrt{C_{2}^{2}-2 C_{1} C_{2} \sin \gamma_{*} \cos \Delta} \sin (\omega t-\omega)
\end{array}\right\}
$$

тде

$$
\begin{gathered}
\operatorname{tg} w=\operatorname{tg} \Delta \frac{1}{1-\frac{1+K_{2}^{2}}{K_{2}} \frac{\sin \gamma_{*}}{\cos \Delta}} \\
K_{2}=\frac{C_{1}}{C_{2}}=K_{1} \frac{\cos \left(45^{\circ}-\gamma_{0}\right)}{\sin \left(45^{\circ}-\gamma_{0}\right)} .
\end{gathered}
$$

С достаточной для практики точностью можно в формуле (40) считать $K_{2}=1$, что дает

$$
\operatorname{tg} w=\operatorname{tg} \Delta \frac{1}{1-2 \frac{\sin \gamma_{*}}{\cos \Delta}} .
$$

Формула (42) совпадает с формулой (21).

Мы рассматривали случай, когда $P_{0}$ и плоскость падения находятся относительно квазиглавных направлений в разных квадрантах. Аналогично получим для случая, когда $P_{0}$ и плоскость падения находятся в одном и том же квадранте, формулу (25).

Интенсивность света при измерении разности хода выражается соотношением

$$
\frac{2 A_{1}^{2}}{C_{2}^{2}}=K_{2}\left(K_{2}-2\right)+1
$$

и в случае $i=55^{\circ}, n=1,5$ составляет не более $0,25 \%$ от максимальной интенсивности света. 


\section{3. Определение изохром}

Подставляя в соотношение (27) выражения колебаний $u$ и $v$ по (11), имеем

$$
\begin{gathered}
A=I^{\prime \prime} B_{1}\left[\sin \left(\alpha_{M}-\beta\right) \cos \left(\alpha_{M}-\beta\right)+\right. \\
\left.+\cos ^{2}\left(\alpha_{M}-\beta\right) \sin \left(\gamma_{0}+\gamma_{*}\right)\right] \sin \omega t- \\
-I^{\prime \prime} B_{2}\left[\sin \left(\alpha_{M}-\beta\right) \cos \left(\alpha_{M}-\beta\right)-\right. \\
\left.-\sin ^{2}\left(\alpha_{M}-\beta\right) \sin \left(\gamma_{0}-\gamma_{*}\right)\right] \sin (\omega t-\Delta) .
\end{gathered}
$$

Из условия минимума квадрата амплитуды колебания (44) по разности фаз $\Delta$ получим условие появления изохром

$$
\sin \Delta=0 .
$$

Следовательно, при наклонном просвечивании в плоском полярископе изохромы также соответствуют разности фаз $\Delta=m \pi(m=0,1,2, \ldots)$.

Аналогично предыдущему легко показать, что интенсивность света на изохроме при $i=55^{\circ}, n=1,5$ составляет менее $0,5 \%$ от максимальной интенсивности света.

\section{IV. ПРИМЕНЕНИЕ КРУГОВОГО ПОЛЯРИСКОПА}

Пусть на модель падает свет с правой круговой поляризацией, который на осях $x$ и $y$ выражается в виде *

$$
x=I \sin \omega t, \quad y=I \sin \left(\omega t-\frac{\pi}{2}\right) .
$$

При преломлении колебания $x$ и $y$ поворачиваются на направления $u$ и $v$, причем

$$
u=I^{\prime} B_{1} \sin \omega t, \quad v=I^{\prime} B_{2} \sin \left(\omega t-\frac{\pi}{2}\right) .
$$

Проектируя колебания $u$ и $v$ на оси $x$ и $y$ и учитывая, что $B_{1} \approx 1$, $B_{2} \approx 1$, имеем

$$
x=I^{\prime} B_{1} \sin \omega t, \quad y=I^{\prime} B_{2} \sin \left(\omega t-\frac{\pi}{2}-2 \gamma_{*}\right)
$$

и после прохождения модели

$$
x=I^{\prime} B_{1} \sin \omega t, \quad y=I^{\prime} B_{2} \sin \left(\omega t-\frac{\pi}{2}-2 \gamma_{*}-\Delta\right) .
$$

После преломления имеем

$$
u=I^{\prime \prime} B_{1}^{2} \sin \omega t, \quad v=I^{\prime \prime} B_{2}^{2} \sin \left(\omega t-\frac{\pi}{2}-2 \gamma_{*}-\Delta\right) .
$$

* Так как при представлении поляризованного по кругу света все направления равноправны, то можем использовать координаты, наиболее удобные с точки зрения решения задачи.

На фиг. 1 показан вид от анализатора к поляризатору. 
Проектируя колебания $u$ и $v$ на оси $x$ и $y$, получим

$\left.\begin{array}{l}x=J^{\prime \prime}\left[B_{1}^{4}+B_{2}^{4} \sin ^{2} \gamma_{*}-2 B_{1}^{2} B_{2}^{2} \sin \gamma_{*} \cos \left(\frac{\pi}{2}+2 \gamma_{*}+\Delta\right)\right]^{1 / 2} \sin \omega t \\ y=I^{\prime \prime}\left[B_{1}^{4} \sin ^{2} \gamma_{*}+B_{2}^{4}-2 B_{1}^{2} B_{2}^{2} \sin \gamma_{*} \cos \left(\frac{\pi}{2}+2 \gamma_{*}+\Delta\right)\right]^{1 / 2} \sin (\omega t-w)\end{array}\right\}$

где

$$
\operatorname{tg} w=\frac{-\cos \left(\Delta+2 \gamma_{*}\right)}{\frac{1+K_{1}^{4}}{K_{1}^{2}} \sin \gamma_{*}+\sin \left(\Delta+2 \gamma_{*}\right)}
$$

Эллиптическое колебание (51), которое обозначаем сокращенно

$$
x=D_{1} \sin \omega t, \quad y=D_{2} \sin (\omega t-w),
$$

можно выразить как сумму двух круговых колебаний, амплитуды которых имеют вид

$$
A_{1,2}^{2}=\frac{1}{4}\left(D_{1}^{2}+D_{2}^{2} \pm 2 D_{1} D_{2} \sin w\right),
$$

где $A_{1}$ - амплитуда правокругового колебания (знак плюс) и $A_{2}$ амплитуда левокругового колебания (знак минус).

Независимо от того, пропускают вторая пластинка в четверть волны и анализатор правокруговое или левокруговое колебание, условие экстремума выходящего из анализатора света имеет вид

$$
\cos w=0 \text {, }
$$

на основе которого имеем из (52)

$$
\frac{1+K_{1}^{4}}{K_{1}^{2}} \sin \gamma_{*}+\sin \left(\Delta+2 \gamma_{*}\right)=0
$$

и далее, учитывая, что $K_{1} \approx 1$,

$$
\sin \left(\Delta+2 \gamma_{*}\right)=-\sin 2 \gamma_{*} .
$$

Из выражения (57) получим условия появления изохром

$$
\Delta=-4 \gamma_{*}+2 m \pi, \quad \Delta=(2 m+1) \pi, \quad(m=0,1,2, \ldots) .
$$

Если же падающий на модель свет имеет левокруговую поляризацию, то условия появления изохром имеют вид

$$
\Delta=4 \gamma_{*}+2 m \pi, \quad \Delta=(2 m+1) \pi .
$$

Из выражений (58) и (59) следует, что в круговом полярископе изохромы могут перемещаться, причем положение изохром зависит кроме $\Delta$ также от угла $\gamma_{*}$ и от вида падающего на модель поляризованного по кругу света *. Однако перемещение изохром малое и при

* При определении направления перемещения изохром относительная разность фаз считается положительной, если при отсчете от плоскости падения против часовой стрелки (рассматривая от анализатора к поляризатору) первое колебание имеет бо́льшую абсолютную фазу, чем второе. 
$i=55^{\circ}, n=1,5$ может составлять максимально $2,5 \%$ от расстояния между смежными изохромами. В целях увеличения точности эксперимента желательно использовать изохромы $\Delta=(2 m+1) \pi$, так как эти изохромы при наклонном просвечивании не перемещаются.

Интенсивность света на изохроме выражается соотношением

$$
\frac{A_{2}^{2}}{B_{2}^{4}}=\frac{1}{4}\left(K_{1}-1\right)^{2},
$$

что при $i=55^{\circ}, n=1,5$ составляет не более $0,25 \%$ от максимальной интенсивности света.

\section{V. ОБ ИЗОБРАЖЕНИИ ВОЗНИКАЮЩИХ ПРИ НАКЛОННОМ ПРОСВЕЧИВАНИИ ОПТИЧЕСКИХ ЯВЛЕНИИ НА СФЕРЕ ПУАНКАРЕ}

Оптические явления, возникающие при прохождении поляризованного света через фотоупругую модель, можно наглядно изобразить при помощи геометрического метода, основанного на применении сферы Пуанкаре $\left[{ }^{5-8}\right]$. Рассмотрим, как этот метод приложим в случае наклонного просвечивания.

На фиг. 4 изображена часть сферы Пуанкаре. Квазиглавные направления обозначены $x$ и $y$, $L-$ плоскость падения. Рассмотрим измерение разности хода компенсатором в плоском полярископе в случае, когда плоскость падения и электрический вектор падающего света находятся относительно квазиглавных направлений в одном и том же квадранте.

По описанной выше методике при измерении разности хода $\mathrm{P}_{0} \mathrm{O} \perp x y$. При преломлении $P_{0}$ поворачивается в направлении плоскости падения на угол $\gamma_{0}$; на фиг. 4 это выражается поворотом на угол $2 \gamma_{0}$ в экваториальной плоскости. $P_{\text {м }}$ разлагается на квазиглавные направления $x$ и $y$. Между колебаниями $x$ и $y$ при прохождении модели возникает разность фаз $\Delta$; на фиг. 4 это выражается поворотом точки $P_{M}$ около диаметра $x y$ на угол $\Delta$. Таким образом, перед выходом из

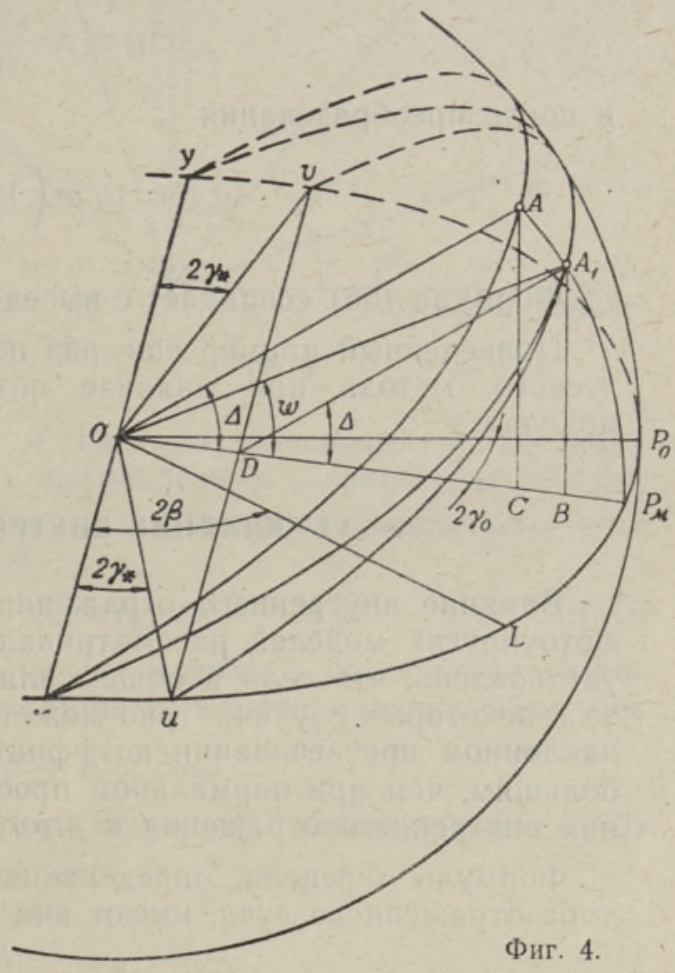
модели световое колебание отображается точкой $A$.

При преломлении колебания $x$ и $y$ поворачиваются в направлении плоскости падения на угол $\gamma_{*}$ (на фиг. $4-2 \gamma_{*}$ ) на направления $u$ и $v$. Разность фаз колебаний $u$ и $v$ также $\Delta$, следовательно, выходящее колебание отображается точкой $A_{1}$, находящейся на плоскости, параллельной плоскости $x A y$ и проходящей через точки $u$ и $v$. Для определе- 
ния измеряемой на осях $x$ и $y$ разности фаз w необходимо определить угол плоскости $x A_{1} y$ с экваториальной плоскостью.

Вследствие малости угла $\gamma_{0}$ можем приближенно считать $P_{\text {м }} O \perp x y$. По фиг. 4 измеряемая разность фаз выражается в виде

$$
\operatorname{ctg} w=\frac{O D+B D}{A_{1} B}
$$

где

$$
\frac{B D}{A_{1} B}=\frac{O C}{A C}=\operatorname{ctg} \Delta
$$

Считая радиус сферы равным единице и учитывая малость угла $2 \gamma_{*}$, можем писать

$$
O D \approx \sin 2 \gamma_{*}
$$

Учитывая, что

$$
A_{1} B=\sin w,
$$

а также соотношение (63), имеем из (61)

$$
\operatorname{ctg} w=\frac{\sin 2 \gamma_{*}}{\sin w}+\operatorname{ctg} \Delta
$$

и после преобразования

$$
\operatorname{tg} \Delta=\operatorname{tg} w\left(1+\frac{\sin 2 \gamma_{*}}{\cos w}\right)
$$

Формула (66) совпадает с выведенной аналитически формулой (26).

Приведенный пример еще раз показывает эффективность геометрического метода при анализе оптических явлений в фотоупругих моделях.

\section{VI. ВЛИЯНИЕ ВНУТРЕННЕГО ОТРАЖЕНИЯ}

Влияние внутреннего отражения при нормальном просвечивании фотоупругих моделей рассматривалось Г. Фавром [9]. В его работе установлено, что если в общем влияние внутреннего отражения мало, то в некоторых случаях * оно может оказаться ощутимым. Так как при наклонном просвечивании коэффициент отражения может оказаться бо́льшим, чем при нормальном просвечивании, то игнорирование влияния внутреннего отражения a priori необоснованно.

Формулы Френеля, определяющие амплитуды электрического вектора отраженного луча, имеют вид

$$
R_{p}=\frac{\operatorname{tg}(i-r)}{\operatorname{tg}(i+r)} E_{p}, \quad R_{s}=-\frac{\sin (i-r)}{\sin (i+r)} E_{s},
$$

где $E_{p}, E_{s}$ - параллельный и перпендикулярный плоскости падения компоненты падающего света; $R_{p}, R_{s}$ - соответствующие компоненты отраженного света.

* Например, при измерении разности хода более точными компенсаторами. 
Обозначаем коэффициенты отражения $\varrho_{p}^{2}$ и $\varrho_{s}^{2}$, тогда

$$
\varrho_{p}^{2}=\left(\frac{R_{p}}{E_{p}}\right)^{2}, \quad \varrho_{s}^{2}=\left(\frac{R_{s}}{E_{s}}\right)^{2} .
$$

В типичном для практики случае $i=33^{\circ}, n=1 / 1,5$ имеем $\mathrm{Q}_{p}^{2}=0,0002 ; \mathrm{Q}_{s}^{2}=0,14$.

Так как $\varrho_{p}^{2} \ll \varrho_{s}^{2}$, будем пренебрегать компонентом отраженного света, параллельным плоскости падения.

Рассматриваем прохождение света через плоскопараллельную пластинку, подвергнутую в общем случае объемному напряженному состоянию с произвольной ориентацией главных напряжений. При этом учитываем кроме первичного луча 1 (фиг. 5) также влияние луча 2 , подвергавшегося дважды (в точках $B$ и $C$ ) внутреннему отражению.

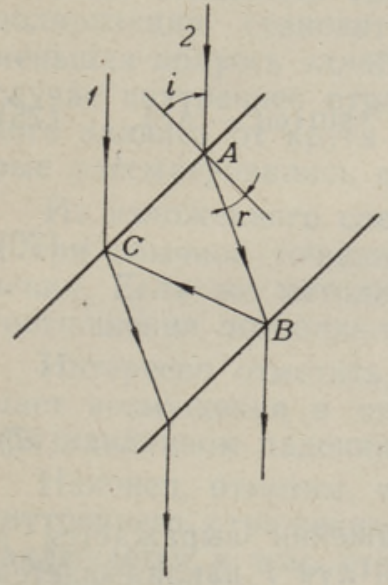

Фиг. 5.

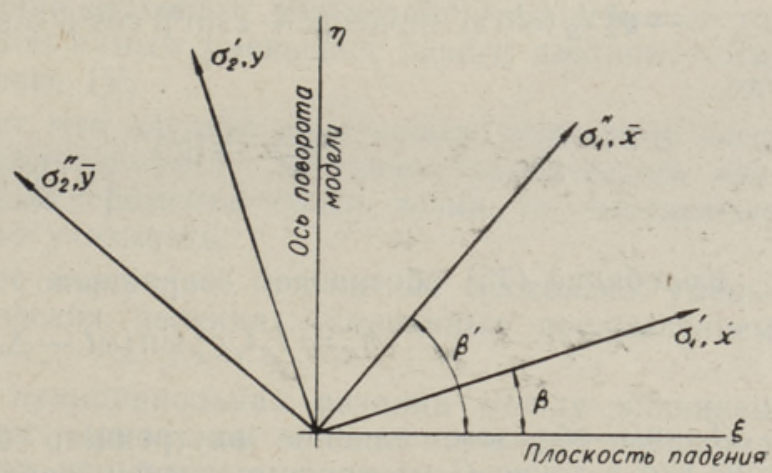

Фиг. 6.

После преломления в точке $A$ свет от плоскополяризованного луча 2 разлагается на колебания в направлениях квазиглавных напряжений для луча $A B-\sigma_{1}^{\prime}, \sigma_{2}^{\prime}$ (фиг. 6). Обозначая амплитуды этих колебаний $I_{x}$ и $I_{y}$, имеем в точке $B$

$$
x_{B}=I_{x} \sin \omega t, \quad y_{B}=I_{y} \sin (\omega t-\Delta) .
$$

После отражения в точке $B$ учитываем только компоненты световых векторов, которые перпендикулярны плоскости падения. Получим

$$
\begin{gathered}
\eta_{B}=\varrho_{s} x_{B} \sin \beta+\varrho_{s} y_{B} \cos \beta= \\
=\varrho_{s}\left(I_{x}^{2} \sin ^{2} \beta+I_{y}^{2} \cos ^{2} \beta+2 I_{x} I_{y} \sin \beta \cos \beta \cos \Delta\right)^{1 / 2} \sin \left(\omega t-\Delta_{B}\right),
\end{gathered}
$$

где

$$
\operatorname{tg} \Delta_{B}=\operatorname{tg} \Delta \frac{1}{1+\frac{I_{x}}{I_{y}} \frac{\operatorname{tg} \beta}{\cos \Delta}} .
$$

Так как в точке $C$ интерференции между лучами 1 и 2 возникать не может, то абсолютная фаза колебания $\eta_{B}$ нас не интересует и можем $\eta_{B}$ обозначать сокращенно

$$
\eta_{B}=\varrho_{s} l_{B} \sin \omega t .
$$


Колебание $\eta_{B}$ разлагается на направления квазиглавных напряжений для луча $B C-\sigma_{1}^{\prime \prime}, \sigma_{2}^{\prime \prime}$. Имеем

$$
\left.\begin{array}{l}
\bar{x}=\eta_{B} \sin \beta^{\prime}=\varrho_{s} I_{B} \sin \beta^{\prime} \sin \omega t \\
\bar{y}=\eta_{B} \cos \beta^{\prime}=\varrho_{s} I_{B} \cos \beta^{\prime} \sin \omega t
\end{array}\right\}
$$

В точке $C$ колебания $\bar{x}$ и $\bar{y}$ имеют разность фаз

$$
\begin{gathered}
\Delta^{\prime}=\frac{2 \pi}{\lambda} C t^{\prime}\left(\sigma_{1}^{\prime \prime}-\sigma_{2}^{\prime \prime}\right) \\
\bar{x}_{C}=\varrho_{s} I_{B} \sin \beta^{\prime} \sin \omega t, \quad \bar{y}_{C}=\varrho_{s} I_{B} \cos \beta^{\prime} \sin \left(\omega t-\Delta^{\prime}\right) .
\end{gathered}
$$

После отражения в точке $C$ имеем

$$
\begin{gathered}
\eta_{C}^{\prime \prime}=\varrho_{s} \bar{x}_{C} \sin \beta^{\prime}+\varrho_{s} \bar{y}_{C} \cos \beta^{\prime}= \\
=\varrho_{s}^{2} I_{B}\left(\sin ^{4} \beta^{\prime}+\cos ^{4} \beta^{\prime}+2 \sin ^{2} \beta^{\prime} \cos ^{2} \beta^{\prime} \cos \Delta^{\prime}\right)^{1 / 2} \sin \left(\omega t-\Delta_{C}\right),
\end{gathered}
$$

где

$$
\operatorname{tg} \Delta_{C}=\operatorname{tg} \Delta^{\prime} \frac{1}{1+\frac{\operatorname{tg}^{2} \beta^{\prime}}{\cos \Delta^{\prime}}}
$$

Колебание (75) обозначаем сокращенно

$$
\eta_{C}^{\prime \prime}=I_{B} I_{C} Q_{s}^{2} \sin \left(\omega t-\Delta_{C}\right) \text {. }
$$

Таким образом, влияние внутреннего отражения выражается в том, что в точке $C$ на преломленный первичный луч 1 накладывается колебание (77).

Наибольшую амплитуду колебание $\eta_{C}^{\prime \prime}$ имеет тогда, когда $\cos \Delta=$ $=\cos \Delta^{\prime}=1$. В этом случае имеем по $(77),(75)$ и $(70)$

$$
\eta_{c}^{\prime \prime}=\varrho_{s}^{2}\left(I_{x} \sin \beta+I_{y} \cos \beta\right) \sin \left(\omega t-\Delta_{C}\right) .
$$

Компоненты результирующего колебания в точке $C$ имеют вид

$$
\xi_{C}=\xi_{C}^{\prime}, \quad \eta_{C}=\eta_{C}^{\prime}+\eta_{C}^{\prime \prime},
$$

где $\xi_{C}^{\prime}, \eta_{C}^{\prime}-$ компоненты преломленного первичного луча 1

$$
\left.\begin{array}{l}
\xi_{c}^{\prime}=\left(I_{x} \cos \beta+I_{y} \sin \beta\right) \sin \omega t \\
\eta_{c}^{\prime}=\left(I_{x} \sin \beta+I_{y} \cos \beta\right) \sin \omega t
\end{array}\right\}
$$

Для амплитуды результирующего колебания $\eta_{C}$ имеем, учитывая,

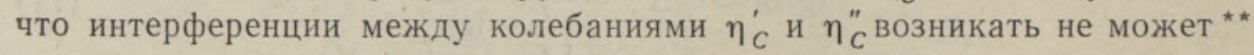

$$
I_{\eta}=\left(I_{x} \sin \beta+I_{y} \cos \beta\right) \sqrt{1+\varrho_{s}^{4}} .
$$

* Как известно, в общем случае объемного напряженного состояния направления квазиглавных напряжений для лучей $A B$ и $B C$ не совпадают.

** Подчеркиваем, что рассматриваем случай, когда отраженный луч имеет наибольшую возможную амплитуду. 
Влияние внутреннего отражения выражается здесь членом $\mathrm{Q}_{s}^{4}$ под корнем. В случае $\mathrm{Q}_{s}^{2}=0,14$ имеем

$$
I_{r_{i}}=1,01\left(I_{x} \sin \beta+I_{y} \cos \beta\right) .
$$

Таким образом, влияние внутреннего отражения выражается в том, что амплитуда колебания, перпендикулярного плоскости падения в точке $C$, увеличивается, причем это увеличение может в случае $i=55^{\circ}, n=1,5$ максимально составлять $1 \%$. Если падающий свет плоскополяризован, то это выражается в повороте электрического вектора преломленного света от плоскости падения. Этот поворот приобретает максимальное значение при $\alpha_{0} \approx 45^{\circ}$ и может при $i=55^{\circ}, n=1,5$ составлять $17^{\prime}$.

Если падающий свет поляризован по кругу, то при преломлении поляризация становится эллиптической [выражения (48)], причем меньшая полуось эллипса перпендикулярна плоскости падения. В этом случае внутреннее отражение несколько уменьшает отклонение светового эллипса от круга и тем самым уменьшает сдвиги изохром, которые рассматривались в главе IV.

Из изложенного следует, что влияние внутреннего отражения мало и при обычной точности эксперимента * не имеет практического значения. Если же методика эксперимента более точна, то выведенные соотношения позволяют это учитывать.

Интересно отметить, что внутреннее отражение несколько уменьшает возмущения в оптических явлениях, вызванных преломлением при наклонном падении.

Наконец, отметим, что принципиальная разница между влиянием внутреннего отражения при нормальном и наклонном просвечивании заключается в том, что при нормальном просвечивании первичный и отраженный луч интерферируют, при наклонном же просвечивании интерференции не возникает. Этим и объясняется то, что влияние внутреннего отражения при нормальном просвечивании гораздо больше.

\section{VII. ЭКСПЕРИМЕНТАЛЬНАЯ ЧАСТЬ}

Некоторые из полученных результатов проверялись экспериментально.

Для проверки формул (21) и (25) использовался растягиваемый образец из целлулоида. На установке КСП-5 при нормальном и наклонном просвечивании определялись параметры изоклин и измерялись разности хода компенсатором Краснова СКК-2 при двух перпендикулярных положениях поляризатора. При наклонном просвечивании $i=55^{\circ}, \beta=43 \div 46^{\circ}, \gamma_{*}=2^{\circ} 07^{\prime}, \quad(n=1,50)$.

На основе формул (21) и (25) можно заключить, что увеличение измеряемой разности хода $w_{1}$ при одном положении электрического вектора падающего света практически равно уменьшению измеряемой разности хода $w_{2}$ при другом положении электрического вектора.

* При рассмотрении внутреннего отражения мы предполагали, что $\varrho_{s}^{2} \gg \varrho_{p}^{2}$. При малых углах падения это условие не выполняется. Однако легко убедиться, что чем меньше угол падения, тем меньше возмущения, вызванные внутренним отражением.

2 TA Toimetised T-2 61 
Поэтому можно считать

$$
\Delta=\frac{w_{1}+w_{2}}{2} .
$$

Результаты эксперимента для случая, когда электрический вектор падающего света и плоскость падения находятся относительно квазиглавных напряжений в разных квадрантах, представлены на фиг. 7.

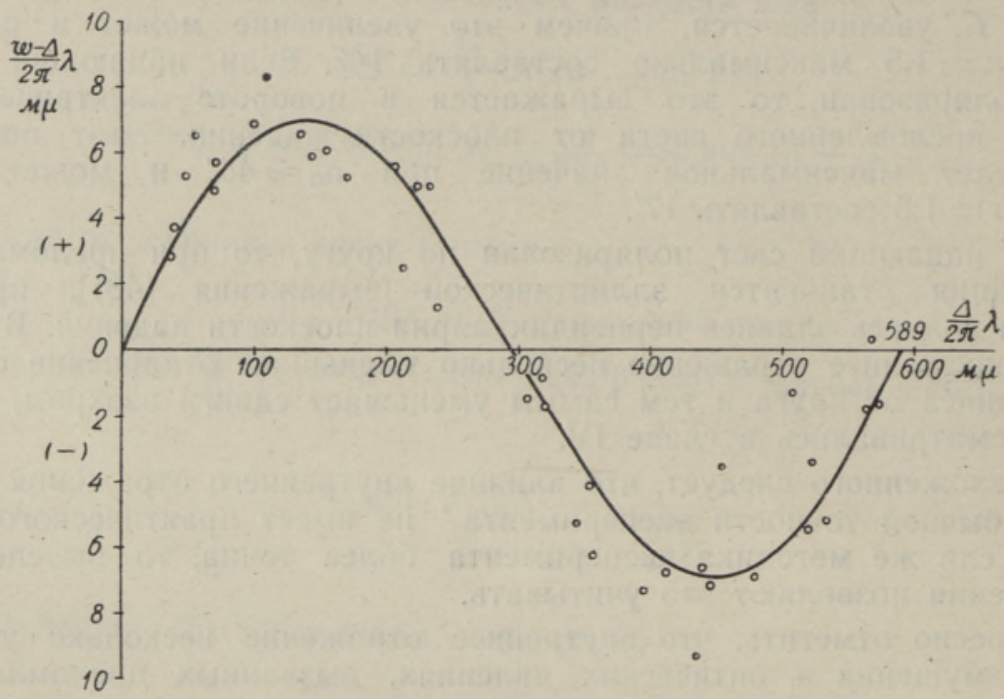

Фиг. 7.

Совпадение экспериментальных данных с теоретической кривой-можно считать удовлетворительным.

При определении изоклин при наклонном просве́чивании наиболь-

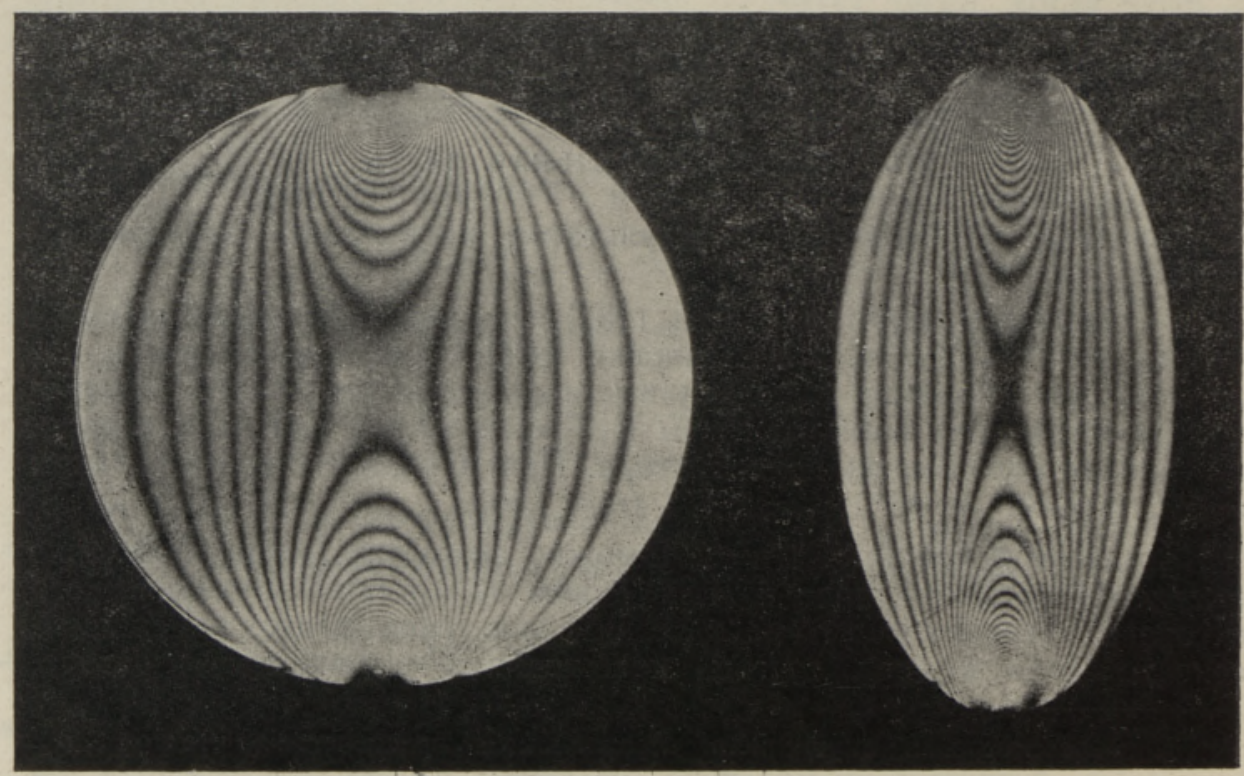

Фиг. 8. 
шее затемнение поля зрения получилось при перпендикулярных друг другу положениях поляризатора (в пределах точности установки КСП-5). Это подтверждает справедливость формулы (35).

Для проверки результатов можно использовать также выведенную в работе [10] формулу

$$
\Delta_{\mathrm{H}} \sin 2 \beta_{н}=\Delta \sin 2 \beta,
$$

где $\Delta_{\text {н }}, \beta_{\text {н }}$ - разность фаз и параметр изоклины при нормальном просвечивании *. При экспериментах условие (84) всегда удовлетворялось в пределах точности эксперимента.

Наконец, в качестве иллюстрации на фиг. 8 приведены фотографии картины полос нагруженного диаметрально сосредоточенными силами круглого диска при нормальном и наклонном просвечивании $\left(i=55^{\circ}\right)$ в круговом полярископе. Диск был изготовлен из эпоксидной смолы диаметром 37,3 мм, толщиной 5,1 мм. Из фиг. 8 видно, что четкость картины полос при наклонном просвечивании почти такая же, как и при нормальном просвечивании.

\section{ЛИТЕРАТУРА}

1. P. A cloque, Méthode optique de mesure et séparation des contraintes en élasticité plane, Compt. Rend. Acad. Sci.. 239, № 21, 1954.

2. В. М. К р а сн ов, О косом просвечивании в фотоупругости, Поляризационно-оптический метод исследования напряжений (труды конференции), Изд. Ленингр. ун-та, 1960.

3. Л. Д. Л анд а у, Е. М. Л и фшиц, Электродинамика сплошных сред, М,. 1957.

4. Handbuch der Physik, Bd. XX, Licht als Wellenbewegung, Berlin, 1928.

5. H. P o i n c a ré, Théorie mathématique de la lumière, II, Paris, 1892.

6. М. Ф. Бокш те й н, Графический расчет поляризационных преобразований, Журнал техн. физики, т. 18, вып. $5,1948$.

7. М. Ф. Б окш т е й н, Геометрический анализ поведения поляризованного света при его прохождении через объемную напряженную модель, Поляризационно-оптический метод исследования напряжений (труды конференции), Изд. Ленингр. ун-та, 1960.

8. R. Plechata, A geometric interpretation of double refraction in photoelasticity, Acta Technica, No. 3, 1957.

9. H. F a vre, Etude théorique de l'influence des réflexions intérieures sur la marche d'un rayon lumineux traversant une lame transparente soumise à des forces agissant dans son plan moyen, Ingenieur-Archiv, Bd. 28, Festschrift Richard Grammel, 1959.

10. Х. А б ен, О применении метода наклонного просвечивания в фотоупругости, Изв. АН ӬССР. Серия техн. и физ.-мат. наук, т. IX, № $1,1960$.

* Формула (84) относится к случаю, когда два главных напряжения находятся в плоскости исследуемой пластинки. 


\title{
OPTILISTEST NĂHTUSTEST FOTOELASTSETE MUDELITE VALGUSTAMISEL KALDKIIRTEGA
}

\author{
H. Aben, \\ tehniliste teaduste kandidaat
}

Resümee

Artiklis vaadeldakse optilisi nähtusi, mis tekivad polariskoobis fotoelastsete mudelite otsesel läbivalgustamisel kaldkiirtega ilma immersioonivanni asetamiseta.

Tööst selgub, et isokliinide määramiseks vōib kasutada ristvalgustamise metoodikat. Kompensaatoriga mōõdetav faasinihe on seotud quasi-peapingete vahele vastava faasinihkega valemite (21) ja (25) abil, kus $\gamma_{*}$ tähistab valgusvektorite pöördenurka murdumisel mudelist väljudes. Valem (21) vastab juhule, mil langeva valguse elektrivektor ja langemistasapind asuvad quasi-peapingete suhtes erinevates kvadrantides, valem (25) aga juhule, mil märgitud suunad asuvad samas kvadrandis. Seega vōib mōōdetav faasinihe olla quasi-peapingete vahele vastavast faasinihkest suurem vổi väiksem (joon. 2). Märgitud erinevus vōib juhul, kui $i=55^{\circ}, n=1,5, \lambda=589 \mathrm{~m} \mu$ ulatuda $7 \mathrm{~m} \mu$-ni.

Tasapolariskoobis tekivad isokroomid analoogiliselt ristvalgustamisele juhul, kui $\sin \Delta=0$.

Tsirkulaarpolariskoobis on isokroomid määratud seostega (58) ja (59), olenevalt sellest, kas langev valgus on parempoolselt vōi vasakpoolselt tsirkulaarselt polariseeritud. Juhul, kui $i=55^{\circ}$ ja $n=1,5$, ei ületa isokroomide nihkumine $2,5 \%$ naaberisokroomide vahekaugusest.

Artiklis vaadeldakse ka murdumisnähtuste käsitlemist Poincaré' sfääril ning tuletatakse geomeetrilisel teel valem (26). Uhtlasi käsitletakse sisepeegelduse mõju kaldkiirtega valgustamisel.

Töö lōpus esitatakse andmed saadud tulemuste eksperimentaalse kontrolli kohta.
Eesti NSV Teaduste Akadeemia
Küberneetika Instiuut
Saabus toimetusse
30. VIII 1960

\section{ON OPTICAL PHENOMENA AT AN OBLIQUE PASSING OF LIGHT THROUGH PHOTOELASTIC MODELS}

\section{H. Aben \\ Summary}

The paper considers the optical phenomena occurring at an oblique passing of polarized light through photoelastic models if these are not placed into an immersion bath.

It is shown that at the determining of the isoclinics the methods of normal incidence are applicable. The phase angle $w$ measured with a compensator is related to the phase angle $\Delta$ which is determined by the difference of the secondary principal stresses, by formulas (21) and (25) ( $\gamma_{*}$ is the angle of rotation of the light vectors by refraction). The formula (21) holds when the electric vector of incident light and the plane of incidence are in different quadrants in relation to secondary principal stresses, the formula (25) in the case when these directions are in the same quadrant. The difference $w-\Delta$, in the case if $i=55^{\circ}, n=1.5, \lambda=589 \mathrm{~m} \mu$ can reach $7 \mathrm{~m} \mu$.

In a plane polariscope fringes occur when $\sin \Delta=0$, as in normal incidence.

If a circular polariscope is used, the fringes are determined by formulas (58) if the incident light is polarized clockwise, and by formulas (59) if the incident light is polarized counterclockwise. In the case if $i=55^{\circ}, n=1.5$ the displacement of fringes does not exceed 2.5 per cent of the distance between adjacent fringes.

The optical phenomena occurring at oblique incidence are analyzed on the sphere of Poincaré, and the formula (26) is derived by geometric methods. The influence of internal reflection at oblique incidence is considered.

Data of the experimental checking of theoretical results are presented.

Academy of Sciences of the Estonian S. S. R., Institute of Cybernetics

Received Aug. 30 th, 1960 